befragt werden. Ob dabei die Zahl von vier Interviewpartnerinnen ausreichend ist, um Rechtstatsachenforschung zu betreiben, kann hier dahinstehen. Repräsentativ ist diese Erhebung bereits deshalb nicht, weil diese sämtlich "better families" entstammten, worauf Glander in anderem Zusammenhang selbst hinweist (S. 129).

Am Ende der Untersuchung steht das Ergebnis "that women in Sana'a do inherit but very often in a way not in line with official prescriptions" (S. 129). Für sich genommen ist diese Schlußfolgerung durchaus plausibel, jedoch folgt dies kaum aus dem von der Verfasserin präsentierten Material. Auch in methodischer Hinsicht dürfte Glanders Hinweis, allein verheiratete Wissenschaftlerinnen seien in der Lage, Feldforschungen im Jemen durchzuführen (S. 18), manche vor den Kopf stoßen. Dies gilt gleichermaßen für ihre "Ten Commandments of Field Research" (S. 14), allgemeinen Verhaltensregeln, wie man sie auch in einem besseren Reiseführer finden kann und die bisweilen eingeflochtenen autobiographischen Schilderungen (etwa S. 97-100). Ob die Verfasserin ihrem Anliegen gerecht wird und mit der vorliegenden Studie zum besseren Verständnis arabischer Gesellschaften beiträgt, kann hier dahinstehen. Denn jedenfalls der Forschungsstand ist nicht ganz so desaströs, wie dies ihre Untersuchung nahelegt (S. 124). So bleibt dem Leser unbenommen, sich an anderer Stelle über Recht und Gesellschaft im Jemen zu informieren. ${ }^{1}$

Kilian Bälz

\title{
Niels-Jürgen Seeberg-Elverfeldt
}

\section{The settlement of disputes in deep seabed mining}

Access, jurisdiction and procedure before the Seabed Disputes Chamber of the International Tribunal for the Law of the Sea

Nomos Verlagsgesellschaft, Baden-Baden, 1998, 166 S., DM 98,--

Eine Monographie über die Streitbeilegung im Tiefseebergbaurecht ist gegenwärtig eine Monographie über einen Streitbeilegungsmechanismus ohne Streit, denn der Abbau von polymetallischen Knollen auf dem Meeresboden außerhalb nationaler Hoheitsgewalt hat aus wirtschaftlichen Gründen immer noch nicht begonnen. Dennoch ist Abschnitt XI des Seerechtsübereinkommens (SRÜ) mit seinen umfangreichen Regelungen diesbezüglich ein faszinierendes Vertragswerk, in dem vor allem der Versuch unternommen wurde, die wirtschaftliche Nutzung von natürlichen Ressourcen völlig neu zu regeln (Prinzip des gemein-

Hingewiesen sei nur auf: Messick, The Calligraphic State. Textual Domination and History in a Muslim State, Berkely, 1993; Mundy, Domestic Government: Kinship, Community and Polity in North Yemen, London 1995; sowie jetzt Wiirth, Ash-Sharî̃a fî Bâb al-Yaman: Recht, Richter und Rechtspraxis an der familiengerichtlichen Kammer des Gerichts Süd-Sanaa, (Republik Yemen) 1983-1995, Diss. FU Berlin 1998. 
samen Erbes der Menschheit, Art. 136 SRÜ). Durch das Übereinkommen zur Durchführung des Teiles XI des Seerechtsübereinkommens der Vereinten Nationen (Durchführungsübereinkommen) von 1994 wurde der Tiefseebergbau jedoch neu geregelt, da sich Teil XI des SRÜ mit seinem, im wesentlichen an den Interessen der Entwicklungsstaaten orientierten Lösungsansätzen als Hindernis für den Erfolg des gesamten Übereinkommens darstellte. Das Durchführungsübereinkommen erfaßt zwar nicht den Streitbeilegungsmechanismus des Tiefseebergbauregimes, jedoch sind durch das Durchführungsübereinkommen völlig neue potentielle Streitfragen entstanden. Damit sind die ursprünglich erwarteten Streitigkeiten unwahrscheinlicher und die daraufhin ausgerichteten Lösungen somit obsolet geworden. Darüber hinaus hat der Internationale Seegerichtshof (ISG) in Hamburg bekanntlich mittlerweile seine Arbeit aufgenommen, am 28. Oktober 1997 eine Verfahrensordnung verabschiedet und damit den Streitbeilegungsmechanismus konkretisiert. Daher ist das anzuzeigende Buch sehr zu begrüßen, und Seeberg-Elverfeldt hat aus gutem Grund seine 1986 vorgelegte Dissertation überarbeitet und in englischer Sprache neu veröffentlicht. Die besondere Bedeutung des Streitbeilegungsmechanismus liegt darin, daß er wesentlich für Erfolg oder Nichterfolg des Tiefseebergbaus verantwortlich ist, denn kein Investor wird die hohen finanziellen Risiken des Tiefseebergbaus auf sich nehmen, ohne sicher sein zu können, daß er seine Interessen rechtlich wirksam schützen und durchsetzen kann. Die Besonderheiten der Streitbeilegung im Tiefseebergbauregime bestehen in der Tatsache, daß es als Teil eines völkerrechtlichen Vertrages obligatorische Streitbeilegung vorsieht, sowie natürlichen und juristischen Personen des Privatrechts Zugang zu einem internationalen Gericht ermöglicht.

In der Einleitung gibt der Autor einen Überblick über die Genesis des SRÜ und zeigt die Probleme im Ratifikationsprozeß sowie die Lösungsansätze durch das Durchführungsübereinkommen auf. Hierbei wird deutlich, daß im Vordergrund der Umgestaltung vor allem die Ausrichtung des Tiefseebergbauregimes an "vernünftigen kommerziellen Grundsätzen" (Abschnitt $6 \S 1$ a) Durchführungsübereinkommen) stand.

Das erste Kapitel stellt detailliert die Entstehungsgeschichte des Streitbeilegungsmechanismus dar, und die Entwicklung von den ersten Überlegungen 1968 bis zur Verabschiedung des SRÜ 1982 wird anschaulich nachgezeichnet. Abschließend gibt der Autor einen systematischen Überblick über die Streitbeilegung im Tiefseebergbau.

Im zweiten Kapitel widmet sich Seeberg-Elverfeldt der Gerichtsverfassung der Kammer für Meeresbodenstreitigkeiten (KSM). Hierbei werden die Errichtung sowie die Funktion der KSM besprochen, wobei die Streitbeilegungskompetenz in drei Gruppen unterteilt wird. Der Verfasser unterscheidet zwischen Streitigkeiten, die zwischen Staaten oder Staaten und Internationalen Organisationen entstehen können. Als zweite Gruppe identifiziert er Konflikte, die zwischen der Meeresbodenbehörde (die Behörde) und den Unternehmen, die Tiefseebergbau betreiben (Vertragsnehmer), entstehen können. Streitigkeiten, die unter den Vertragsnehmern entstehen können, werden schließlich als dritte Gruppe beschrieben. Weiter setzt er sich ausführlich mit den Anforderungen an die Richterinnen und Richter der KSM auseinander. Er erläutert, wie die Zusammensetzung der Kammer unter Berücksichti- 
gung der Vertretung der hauptsächlichen Rechtssysteme der Welt und einer gerechten geographischen Vertretung erfolgt. Kritisch sieht der Verfasser die nur drei Jahre währende Amtszeit, und es ist ihm zuzugeben, daß, obwohl eine Wiederwahl möglich ist, dieser kurze Abschnitt wahrscheinlich einen negativen Effekt auf die einheitliche Interpretation und Anwendung des Rechts haben wird (S. 57).

Das dritte Kapitel behandelt den Zugang zur KSM und legt die allgemeinen Voraussetzungen für die in Frage kommenden Parteien (Staaten, Internationale Organisationen, die Behörde, natürliche und juristische Personen) dar. Es wäre wünschenswert, wäre an dieser Stelle auch neuere Literatur berücksichtigt worden, die sich mit der Entwicklung des Einzelnen als Partei in internationalen Streitigkeiten auseinandersetzt. Im allgemeinen ist unklar, warum der Verfasser nicht die gegenwärtige Literatur stärker einbezogen hat und nach wie vor Literatur aus den Auflagen zur Zeit der ersten Veröffentlichung seiner Dissertation 1986 zitiert.

Das vierte Kapitel bildet den Schwerpunkt der Arbeit und stellt umfangreich die Zuständigkeiten der KSM dar. Hier wird nochmals deutlich gemacht, daß das SRÜ im Tiefseebergbau zwar obligatorische Streitbeilegung vorsieht, daß die KSM aber keine ausschließliche Zuständigkeit für Streitigkeiten in diesem Bereich hat und den Parteien die Wahlmöglichkeit bezüglich des .Spruchkörpers bleibt (Art. 188 (1) und (2) i.V.m. Art. 187 a) SRÜ). Neben der KSM kann daher eine Streitigkeit durch eine Sonderkammer des ISG, eine Ad-hoc-Kammer der KSM oder durch ein bindendes Handelsschiedsverfahren beigelegt werden. Allerdings behält die KSM die Zuständigkeit über Fragen, die die Auslegung des Teiles XI des SRÜ und der sich darauf beziehenden Anlagen sowie des Durchführungsübereinkommens berührt. Das Handelsschiedsgericht muß entsprechende Auslegungsfragen der KSM vorlegen. Im weiteren geht der Autor auf die sich abzeichnenden Probleme bei der Arbeit der KSM ein, die sich aus der Begrenzung der Zuständigkeit der KSM hinsichtlich der Beschlüsse der Behörde ergeben (Art 189 SRÜ). Besonders treten die mangelhaften Rechtsschutzmöglichkeiten hervor, die sich für Private daraus ergeben, daß die KSM keine Ermessensentscheidungen der Behörde überprüfen kann (Art. 189 Satz 1 SRÜ; S. 93). Darüber hinaus kann die KSM nicht über die Vereinbarkeit von Regeln, Vorschriften oder Verfahren der Behörde mit dem SRÜ entscheiden oder diese für ungültig erklären (Art 189 Satz 2 SRÜ). Die starke Stellung der Behörde erklärt Seeberg-Elverfeldt damit, daß rechtsetzende Entscheidungen der Mehrheit der Staaten nicht durch ein Gericht sollen aufgehoben werden können. Selbst in dieser modern anmutenden Kodifikation zeigt sich damit wieder der eher rudimentäre Charakter des Völkerrechts, denn was seit Marbury vs. Madison (Oberster Gerichtshof der Vereinigten Staaten vom 24. Februar 1803) innerstaatlich in den meisten demokratischen Staaten anerkannt ist, hat im Völkerrecht noch einen langen Weg vor sich: Die gerichtliche Kontrolle von Normen anhand übergeordneten Rechts. Der Verfasser resümiert, daß der Mangel an angemessenem rechtlichen Schutz für private Unternehmer gegenüber der Behörde eine wesentliche Schwäche des Rechtsschutzsystems des SRÜ darstellt (S. 115). In diesem Kapitel werden schließlich die Vorausset- 
zungen für die Haftung der Behörde für rechtswidriges Verhalten erläutert, wobei Art. 187 e) SRÜ die Rechtsgrundlage für ein Verfahren vor der KSM gegen die Behörde ist.

Am Ende der Arbeit setzt sich Seeberg-Elverfeldt mit Verfahrensfragen der KSM auseinander (Kapitel 6). Hierbei treten zwei Fragen in den Vordergrund. Zum ersten gibt es für Klagen vor der KSM weder im SRÜ, dem Statut des ISG, noch im Durchführungsübereinkommen oder in der neuen Verfahrensordnung des ISG Klagefristen. Dieser Befund erhöht die Rechtsunsicherheit und kann zu verfahrensrechtlichen Problemen in der zu erwartenden Praxis vor der KSM führen. Der Vorschlag des Autors zur Lösung dieses Defizits lautet, die Klagefristen des Gerichtshofs der Europäischen Gemeinschaften (EuGH) zu übernehmen. Als weitere Schwäche der Verfahrensordnung wird die ungeklärte rechtliche Stellung von Dritten im Verfahren benannt. Insbesondere ist unklar, wie Staaten, die einen Vertragsnehmer befürworten (sponsoring states), im Verfahren auftreten.

Die vorliegende Arbeit gibt einen umfangreichen Einblick in die Entstehung, das Verfahren und die zu erwartenden Probleme im Streitbeilegungsmechanismus des Tiefseebergbauregimes. Dem Autor ist es gelungen, die Materie so auf zubereiten, daß zukünftige Investoren einen educated guess wagen können, was sie bei Streitbeilegungsverfahren in der $\mathrm{Zu}-$ kunft erwartet. Zuzustimmen ist Wolfrum, der die Besprechung der deutschen Ausgabe (GYIL 34 (1991), S. 572) mit der Bemerkung beendet: "In der Praxis - sollte es zu dieser kommen - wird die Arbeit zum Einstieg in die Praxis des Seegerichtshofs von großem Nutzen sein". Nun ist der Seegerichtshof mittlerweile errichtet, es bleibt abzuwarten, wann der Tiefseebergbau Wirklichkeit wird.

Bernhard Braune

\section{Johannes Enno Harders}

\section{Regionaler Umweltschutz in der Arktis}

Die völkerrechtliche Pflicht zur Zusammenarbeit und die Drittbindungswirkung eines Regionalmeerabkommens

Nomos Verlagsgesellschaft, Baden-Baden, 1997, 262 S., DM 69,--

Alfred Andersch greift im Nachwort zu "Hohe Breitengrade" ein eigentümliches Wort auf, mit dem die norwegische Sprache die von der Arktis ausgehende Faszination beschreibt, der besonders Wissenschaftler immer wieder erliegen. Sie nennt diese Leute "arktis-bitten", "von der Arktis gebissen", also "von ihr in den Bann gezogen". Johannes Enno Harders, ein solches "Arktis-Opfer", hat mit der vorliegenden Studie, die 1995 der Universität Frankfurt a. M. als Dissertation vorlag, auf dem dürren Feld der deutschsprachigen völkerrechtlichen Literatur zum Thema Arktis (und zum arktischen Umweltschutz im besonderen) neues Grün sprießen lassen. Anders als im Südpolarraum fehlt in der Arktis ein übergreifendes vertragliches Schutz- und Nutzungsregime. Dies macht die Arktis in völkerrechtlicher 